\title{
A Concept for Context-aware Multihoming with Heterogeneous Radio Access Technologies
}

\author{
Stefano Carrella, Christian Mannweiler, Andreas Klein, Joerg Schneider, and Hans D. Schotten \\ Chair for Wireless Communications and Navigation \\ University of Kaiserslautern, Germany \\ Email: \{carrella,mannweiler,aklein,schneider,schotten\}@eit.uni-kl.de
}

\begin{abstract}
There are several radio access technologies providing connectivity for mobile devices. In the future, more and more mobile devices will be capable of supporting more than one access technology. For being able to use these technologies simultaneously, there is a need for multihoming capabilities. Due to the variety of access technologies and the ability to use them simultaneously, new concepts for managing multihoming are needed. Higher requirements on efficiency and user satisfaction put new challenges on decision processes for multihoming. These higher requirements can be met by incorporating context information in the decision process. To enable context-based decisions, there is the need for determining, acquiring, and managing the required context information as well as the need for a decision scheme facilitating this information.
\end{abstract}

\section{INTRODUCTION}

Mobility aspects are getting more and more important in every day life. Communication must keep up with this increasing level of mobility. Due to technological advancements and high user requirements, an integration of existing access technologies is desirable. An integration may also help to achieve a more efficient use of existing network resources. One concept enabling this integration is multihoming. Multihoming allows to simultaneously have more than one connection on one terminal. In an IP-based system, this means to use a set of IP addresses instead of only one. When having the possibility of maintaining more than one connection, there is the need for deciding with which set of networks to connect and which connection to use as primary connection. For each application, there may be another primary path. While in a single-homed terminal, there is just the need to choose the best connection, in a multi-homed terminal, the decision process is much more complicated. The best decision regarding the connections depends on several parameters. These parameters can be seen as context of user and his terminal on the one side and context of the network on the other side. When designing a system being able to make decisions concerning possible connections, it is helpful to integrate the context information as basis for the decision process. For this purpose, a context management system for acquiring, processing, and delivering context information is required.

The concept presented in this paper discusses a method to acquire, manage, and use context information for decisions in multihoming. Chapter 2 presents related work in the area of multihoming and context management. Chapter 3 presents the concept of context-aware multihoming. In a first step, the information needed must be determined. The required information are listed and discussed in chapter 4 . Chapter 5 illustrates how to manage this information in a context management system. Based on this information, system decisions can be made. This decision process is discussed in chapter 6. Chapter 7 concludes this paper.

\section{RELATED WORK}

Multihoming and context awareness have been topics of several research projects. For future wireless networks, multihoming should be enriched with context awareness. The concept presented in this paper uses context information for the decision processes in multihoming. As a basis, related work in the fields of multihoming and context awareness will be presented in the following paragraphs.

\section{A. Multihoming}

The future of wireless communications will comprise several different access technologies. Mobile devices will support multiple radio network interfaces (e.g. WLAN and UMTS). For being able to exploit the variety of technologies, an efficient way for handling these connections must be found. One major aspect is being able to use these access technologies simultaneously, also referred to as multihoming. In an IP-based system, it means supporting more than one IP-address. So there is a need of protocols that can enable handling several IPaddresses simultaneously and additionally enable the section of the best connection for mobile devices.

Research topics on Loc/ID split (location identifier split) and mSCTP (mobile Stream Control Transmission Protocol) aim to find solutions for an efficient way to handle more than one connection in a heterogeneous network landscape.

Loc/ID split enables to have one identifier for the host, independent of the used connections. So there is the possibility to switch between existing physical connections and to set up new physical connections for each host without disruption of the logical connection. With this concept, mobility can be achieved and the transport layer is only aware of one single IP address. But there are some drawbacks in routing. The mapping system must be updated fast enough and the IP addresses must be routable in visited domains. [25]

mSCTP enables to add and remove IP addresses dynamically. The primary path can be set dynamically as well. This dynamic way to handle IP addressees enables multihoming 
for mobile terminals [20], [27]. In addition to the possibility of dynamic IP address configuration, there is the need to enable seamless handovers. Only by having the combination of dynamic IP address configuration for multiple IP addresses and seamless handovers, a seamless connectivity in multihoming can be guaranteed.

There are already existing concepts discussing seamless handover for multi-homed terminals. [24], [26] describe dual-homing (WLAN/UMTS) using mSCTP. [21] presents a method for fast handover based on mSCTP using FMIPv6. In these papers, the handover process itself has been analyzed. However, for an efficient use of multihoming, there is also the need to provide context information on which an optimal selection of idle and active connections can be based on.

For selecting the connections to be established and for setting the primary path, information about available networks and user preferences are needed. All these information can be acquired and delivered by a context management system.

\section{B. Context Awareness}

The notion of context awareness can be found in manifold variations in different research areas of computer science. On the one hand, context consumers, such as context-aware applications and services, require context information in order to adapt to changes in their environment. On the other hand, context providers, e.g. sensors and (wireless) sensor networks, are key components for detecting the requested context. Moreover, GPS modules, temperature, ambient light, and acceleration sensors are becoming widespread with the recent success of smartphones and other mobile devices. For categorization of context sources, Chen [9] proposes the following classes:

1) Direct access to sensors - Sensors deployed on network terminals can collect environmental context such as location or temperature as well as network context information. These sensors are complemented by wireless sensor (and actuator) networks that are installed in a given location. Context providers either have access to these context sources or they physically encapsulate sensors. For instance, a mobile terminal can be the source of context as well as act as a context providing entity within a context management system.

2) Middleware infrastructure - The introduction of middleware infrastructure aims at strictly separating the processes of context acquisition and context management. The separation improves system extensibility and reusability. Conceptionally, a separation between a context providing entity and a context source is based on the middleware approach.

3) Context server - In this approach, a resource-rich context server takes over the task of administering context data that it receives from various context sources. It relieves sensors and terminals from managing context requests from other entities. The server can be accessed by multiple entities for context retrieval without compromising system stability. In addition, the server could also perform reasoning activities, i.e. to infer higher level context data (e.g. weather) from raw sensor data (e.g. temperature, humidity, and light conditions).

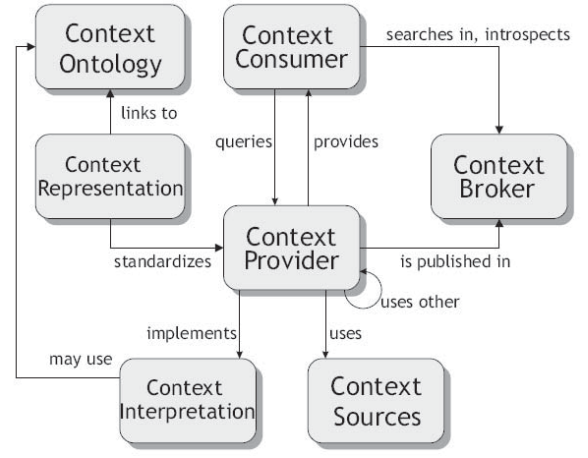

Fig. 1. MobiLife Context Management Framework

Context management systems acquire, process, manage, and distribute context information according to the specific needs of applications and services. Exploiting the available context information for improving multihoming management is a meaningful application beyond conventional end consumer services such as location-based services. However, in both cases, a context management system is required in order to properly supply the application with context, thus making it "context-aware". Many of the early context-aware systems in science have just been location-aware. The Active Badge Location System by Want et al. [10] was one of the first context-aware applications. The current location of a user was determined using infrared-based techniques. In the 1990s, location-aware tourist guide systems were developed that replaced or complemented traditional tourist guides [11], [12], [13]. Meanwhile, there have been improvements with regard to both architectural frameworks for context-aware systems and the diversity and quality of available context information (e.g. advanced positioning technology [14]). European projects such as SPICE [15] and MobiLife [16] have set milestones in integrating context awareness functions into their respective architectures. Figure 1 depicts the MobiLife context management framework with its split into relevant functions. Other authors, such as Chen [9], have proposed agent-based approaches. However, all of these frameworks have often been designed according to requirements of endconsumer services, e.g. location-based services, whereas the proposed context management system can also cope with the requirements of multihoming systems. Furthermore, they miss a comprehensive concept for the consideration of context quality.

\section{Concept of Context-Aware Multihoming}

The availability of multiple access technologies that can be used simultaneously provides ubiquitous connectivity and a better utilization of network resources. For taking advantage of these technological advancements there is a need for being able to switch between these radio access technologies dynamically, depending on the user's situation and network conditions. For that reason, context information should be added in the decision process. The selection of the best connection depends on this information. Even though there are many 
concepts for either multihoming or context management, there are few concepts applying context information to decision processes in multihoming (e.g. [22] and [23]).

These decision processes aim at reaching objectives of the network as well as those of the terminal. From a network operator perspective, an efficient use of network resources and financial interests are relevant. The efficient use of the network resources consists of high data throughput and satisfaction of a high number of users. For that reason, load balancing, reduced signaling overhead, and a small number of handovers are sub-objectives. For reaching these objectives, the decision process can be located at an instance in the network. In this case the network must be aware of all relevant context information of all subscribers to allocate the network resources in an optimal way. Due to different network operators, a huge amount of time variant context information, and privacy issues, the decision process should not be located in the network. So in the concept proposed in this paper, the context management system and the decision process are located in the terminals.

From a terminal perspective, high QoS, high security and privacy, low power consumption, and cost issues are dominant criteria. The QoS dimension consists of the criteria high average data rate, high minimum guaranteed data rate, seamless connectivity, low delays and jitter, and no disruption. This can be aggregated in the expression ABC ("always best connected").

In multihoming, $\mathrm{ABC}$ consists of an optimal combination of ideal connections and used primary path. This combination depends on the applications running on the terminal. For instance, conventional Internet surfing imposes less security and privacy requirements than Internet banking.

In [22] as well as in [23] the decision process is made by the terminal. In these terminal-centric approaches, there is the problem that the terminals can make their decisions only based on their interests and ignore network objectives. To avoid such behavior, users can be charged for services so that the terminals, by including a cost function in their decision processes, implicitly consider network objectives. Another way is to include the criterion bandwidth consumption in the decision process. In this case, there is the risk that the terminal does not implement this component if there is no mechanism for punishing the terminal.

The concept of this paper follows a terminal-centric approach. Network objectives are represented by the prices a terminal has to pay for using the network. This terminal-centric approach considers context-aware multihoming beginning with the acquisition of context information, then managing this information and at least the decision making processes.

First, the required context information for reaching the above mentioned objectives must be determined. [22] uses the following criteria for deciding which path to use: cost of service, security, power consumption, network conditions, and network performance. [23] considers the criteria signal strength, data rate, and bandwidth utilization. While high signal strength and high data rate lead to a better rating of a connection, high bandwidth utilization is evaluated negatively because of the diminution of available bandwidth for other subscribers. So the first and the second criterion consider

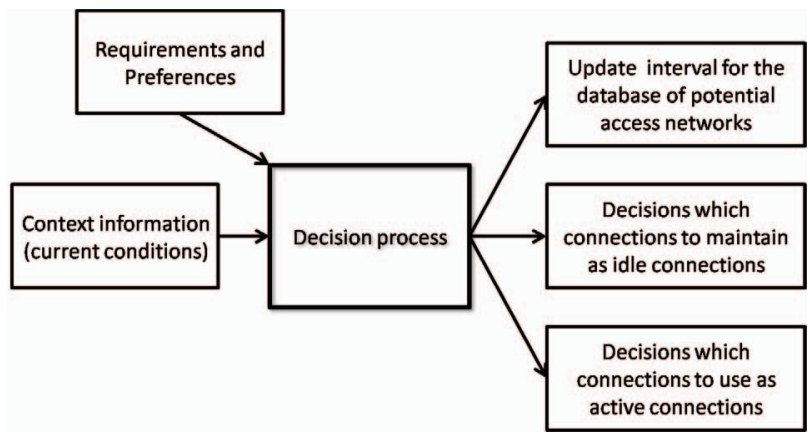

Fig. 2. Concept of Context-awareness for Multihoming

the interests of the individual terminals, the third criterion considers the interest of the operator in a good network performance.

The concept proposed in this paper extends [22], [23] by using a much wider spectrum on context information, in particular by additionally focusing on location and movement. Information about the availability of the network as well as the network conditions must be used for building a model of the network on which decisions can be based. User preferences are then used to determine the best combination of connections. All this information not only must be provided but also managed. A closer look on providing and managing context information is given in chapter 4 and 5 .

By having this context information, decisions can be made to reach the objectives more precisely. Due to the fact that each terminal may be able to support various connections, the combination of off-state, idle, and active interfaces must be predefined. Based on this decision, there is further the need to decide

- which connection to set up,

- which connection to release, and

- which connection to use as primary path depending on each session.

The decision process is described in detail in chapter 6 .

Figure 2 gives an overview over the decision process. Context information consisting of the current conditions and of the requirements are the input for the decision. A set of potential networks, a set of idle connections, and a set of active connections as well as the update period are the output of the decision process.

\section{REQUIRED CONTEXT INFORMATION}

In a first step, the required context information for the decision process must be determined. Context information can be subdivided in five categories:

- availability of networks,

- location and movement of the terminal,

- QoS of available networks,

- security and privacy issues, and

- costs and battery consumption.

All available networks must be determined. For having an anticipatory component, not only information about current availability but also about prospective availability are relevant. 
For this purpose, location and coverage area of the points of attachment (e.g. access point or node B) on the one hand and location and technological capabilities of the mobile terminal on the other hand must be known. By having information about the points of attachment, a virtual map of the wireless landscape can be generated. Knowledge about the network can be acquired by scanning for networks or by communicating with one single point of attachment over an existing connection. The first approach leads to a high energy consumption and potential disruptions of the current connections. In the second approach the terminal depends on the information provided by the point of attachment. In case of different network operators, the requested information can be incomplete or even intentionally not be forwarded to the mobile node, due to conflicting financial interests of different network operators. So each way to find out potential connections has its own drawbacks.

Since there are and will be terminals not supporting every access technology, the first step is to verify whether the mobile terminal is able to support a connection via existing access networks with its technological capabilities. A further criterion is the location of the mobile node. Only if the location of the mobile terminal is and will be in the coverage are of the potential point of attachment, a connection can be set up and used. For predicting the future location, the current movement and movement prediction are needed. For providing the information about the current location, GPS, triangulation, cell ID, etc. can be used for determining or estimatig the location. Additionally, more abstract context information such as postal addresses can be incorporated. To predict the future location, the movement of the mobile node must be used for a location prediction. Due to the fact of not knowing the future movement, a movement prediction can be performed based on the current movement and the context of environment such as street layouts and arrangement of buildings.

After a set of relevant access networks has been determined, each network must be evaluated based on a set of criteria. One criterion is QoS. QoS can be subdivided in average data rate, minimum guaranteed data rate, maximum and average delay, jitter, probability of interruption, possibility of seamless handover, and amount of packet loss. These sub-criteria of QoS are used for evaluating each potential connection. The criteria can be measured, or extracted from a database. A measurement usually has the advantage of a higher significance, while the extraction of the database leads to less traffic and power consumption. A combination of both can also be used, e.g. the database information for a pre-selection and the measurement for a final selection, based on the already reduced set of potential networks. Which kind of data should be used for the selection and also the weight of each criterion can further depend on the requirements of the applications running. The criterion signal strength should not be used for the evaluation of QoS. Criteria of higher levels should be used instead, e.g. data throughput. For instance, even when the received signal strength from a specific point of attachment is very good, the data rate can be low, caused by a high number of users.
Security and privacy issues are important as well. Depending on the application, different security and privacy requirements must be met. These criteria are used as knockout criteria. Only the networks that satisfy these requirements should be used.

The user is not only interested in QoS, security, and privacy, but also in spending less money and saving battery power. So the criteria costs and power consumption are incorporated as well.

Further, AAA possibilities must exist, so that the capability of the network and existing contracts also can be taken into account. However, in the proposed concept, AAA criteria are not further considered because of the low technical relevance.

\section{A Context Management System FOR MUltihoming ARCHitectures}

Ideally, context management systems should be agnostic with respect to their application. However, depending on the intended utilization of context information, context management concepts have to comply with different requirements. In this section, we propose a context management architecture (CMA) that is based on the producer-consumer role model that can frequently be found in the area of context management, e.g. [19]. The CMA is designed to acquire, manage, and distribute context information and to control the context quality required for multihoming purposes. This functionality requires the following core architectural components:

- Context Provider

- Context Broker

- Context Consumer.

\section{A. Context Provider}

The Context Provider (CP) is the logical point in the CMA where context information originates from and is provided to other entities of the architecture. The types of context data a CP provides can be classified in (relatively) static data on the one hand and dynamic data on the other. Static data for instance include terminal capabilities, user preferences, or user information retrieved from social communities on the web. However, for the purpose of multihoming, dynamic context information, such as user location and movement (i.e. speed and direction) and network conditions have a higher relevance. Additionally, functional modules that apply reasoning algorithms on low-level context data are able to abstract and predict user behavior, e.g. user movement, thus acting as CPs for higher-level context. The communication between CPs and other CMA entities occurs in a synchronous mode, i.e. CPs directly reply upon context requests. For initial advertising of its capabilities and availability to the architecture, the $\mathrm{CP}$ sends an announcement containing the respective information to the Context Broker.

\section{B. Context Broker}

The Context Broker (CB) takes on the role of a middleman. Its main functionality is to maintain a registry of available $\mathrm{CPs}$ and their capabilities based on the announcement sent by CPs. 
Based on that registry, it can provide a CP look-up service to entities searching for certain context data. In addition, the $\mathrm{CB}$ can provide context data itself by forwarding the data it has received from CPs. Two different communication modes are available for requesting entities. In the asynchronous mode, context is forwarded if a specified condition or event comes true ("publish/subscribe mode"). In the synchronous mode, a request for context information is instantly answered by the CB ("request/provide mode"). In order to allow for forwarding of context, the $\mathrm{CB}$ itself maintains a context cache where it stores context that has not expired yet. Expired context is moved to the context history database where it can be accessed by the $\mathrm{CB}$ if necessary.

\section{Context Consumer}

Context Consumers (CC) are entities that use context data as an input for their actual functionality. Network services, applications for end users, service enablers or actuators in wireless sensor and actuator networks are exemplary consumers of context information. The $\mathrm{CC}$ we focus on in this paper consists of the multihoming management system. Generally, CCs can request context from the $\mathrm{CB}$ or they can invoke an URI of a CP at the broker. Consequently, CCs can establish a direct communication with the according $\mathrm{CP}$.

\section{Overall Architecture}

Figure 3 shows how the entities presented in the previous sections are integrated into the CMA. The main part of communication between a $\mathrm{CC}$ and a $\mathrm{CP}$ takes part via the $\mathrm{CB}$. However, $\mathrm{CCs}$ have the possibility to request the URI of CPs at the broker (CP look-up service). Using this URI, they can communicate directly to a $\mathrm{CP}$ and request context data, thus speeding up context data delivery. Two different communication modes are utilized for enabling the described functionality. The synchronous mode implements a "request/provide" scheme. A Context Consumer can send a context query to a providing entity which in turn will immediately respond to this query by transmitting the respective context data. In contrast, the asynchronous mode is based on an event-driven "publish/subscribe" scheme. In this case, a Context Consumer can specify a condition (e.g. elapsed time span or signal level below threshold) upon which it wants to receive a specific context from the providing entity. As soon as this condition is satisfied, the providing entity will publish the requested data to the respective consumer. The conceptual outline of the architecture allows for controlling context information in a scalable and extensible way.

\section{DECISION PROCESS}

Every mobile terminal has a database containing information about potential, idle and active connections. Potential network connections are those that can be established at the current and at the very probable future location. Using this information, a new connection can be set up much faster. Idle connections are needed for being able to switch fast to an active status. It would be too time consumptive to set up a

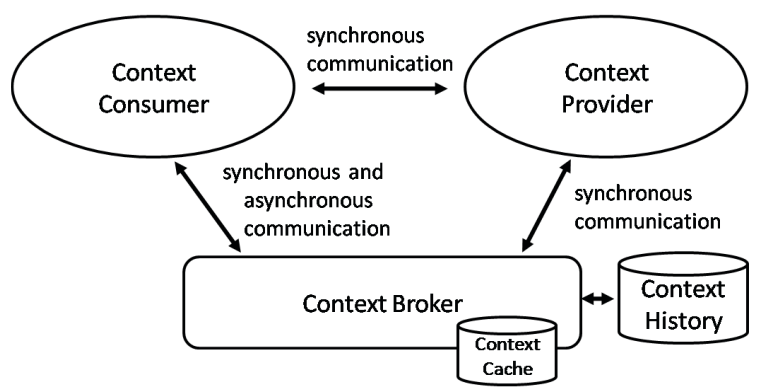

Fig. 3. Context Management Architecture

new connection when needed. Only active connections can be used for communication. Each application can use a different connection and so a different network. Which connections should be in an idle and which in an active state must be determined in a decision process. This decision process is based on the information provided by the context management system. The management of the database containing the potential connections depends on some context information as well (e.g. location).

For updating the database, two criteria are used in this paper: a timer and user location. The decision process is depicted in figure 4. If a certain time has expired or if the mobile node has changed its location, the database is updated. If the mobile terminal only has few potential access networks in its database, updates should be performed more frequently. As contribution to the fact that there are different access technologies with different characteristics, the time of updates for each access technology should depend on the characteristics of the technology itself.

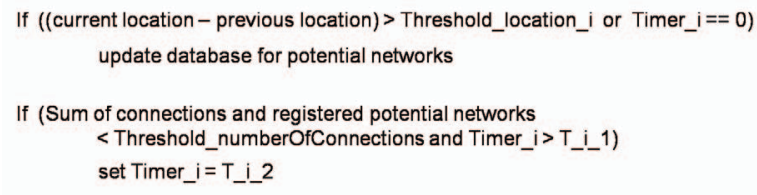

Threshold_location_i: For each technology i there is a threshold value for the location change Timer_i. For each technology $i$ there is a timer starting with T_i_1 and counting down to 0 T_i_1: Start value of the timer for technology $i$

T_i_2: Modified timer value for having a more frequent update of potential access networks 2. Modified timer value for having a more

Threshold_numberOfConnections: Desired minimum number of potential access networks in the data base

Fig. 4. Database Update Scheme

If battery power is low, Threshold_location, and the timers, $\mathrm{T} \_\mathrm{i} \_1$, and $\mathrm{T} \_\mathrm{i} \_2$, respectively, are set to a higher value for reducing updates and saving power.

The criteria characterizing each potential connection including their sub-criteria

- supported QoS class

- supported Security and Privacy class

- Coverage area and used access technology

are stored in the database. Context information, such as delay and jitter, can be requested at a current point of attachment 
using an active connection. This point of attachment can provide the information by first communicating with other potential points of attachment. So no measurement is needed. By using the point of attachment for acquiring the information and using already stored information, signaling overhead can be reduced and the quality of the information is sufficient for characterizing the potential connections and for a pre-selection of idle connections.

Active connections that are not longer needed to be in an active state will be transferred to an idle state. In addition, out of the set of potential access networks, a set of idle connections can be selected. The criteria location and movement are used in the first step for deciding which connection should be in an idle state for being able to activate it immediately. By predicting future location, suitable access networks can be selected. The faster the movement, the more important are connections to radio access networks with large coverage areas.

After the pre-selection based on location and movement, further criteria are needed for selecting the best set of connections. The set of idle connections should meet the criteria high QoS and high privacy and quality so that the user is enabled to start a connection with any requirements just by activating an idle connection without delay caused by setting up a completely new connection. In addition, if there already is an application running on the terminal, at least one idle connection should be suitable as back-up connection and meeting the requirements of the application. In case of interruption of the active connection, the idle connection can be activated immediately.

Based on the above mentioned criteria, the set of connections is selected. While in the case of potential access networks, no measurements of QoS is needed, the idle connections should be activated from time to time and a measurement of QoS should be performed for having a more precise and more up-to-date decision base. Similar to the refreshment of the database for potential connections, the set of idle connections should be renewed.

Active connections should only be maintained while needed. Each application should be able to select the connection best suited for its requirements. While communicating, a new connection can be selected. For that reason, the selection of the active and used connections must take location, movement, and handover capabilities for each connection into account. The decision process for the active connections is depicted in figure 5. Depending on user preferences and requirements of running applications, the criteria for selecting the active connection are weighted differently. The overall rating value of each connection is the sum over the weighted values.

Security and privacy issues are knock-out criteria. Only these connections able to meet security and privacy requirements can be used. Out of the set of connections that meet security and privacy requirements, a rating is build based on the following criteria: QoS, handover capabilities, location and movement as well as power consumption. Location and movement are used for determining the handover probability. The value of $g(\mathrm{QoS})$, the weight of the QoS in the rating, depends on the QoS requirements. If the requirements of QoS are met, the weight of QoS is low, with the consequence that

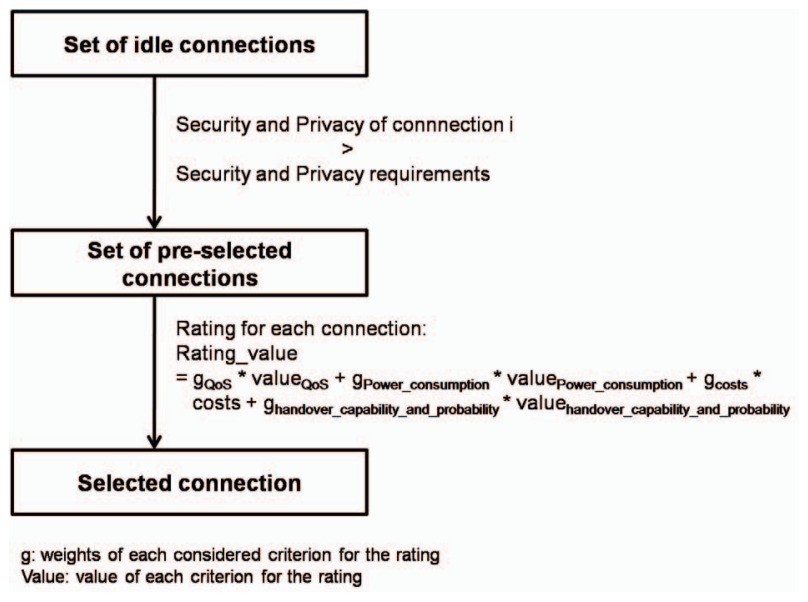

Fig. 5. Selection Scheme for Active Connections

as soon as the QoS requirements are met, the weight of other criteria, in relation to QoS criteria, will be higher. Due to the fact that QoS consists of several parameters, $\mathrm{g}(\mathrm{QoS})$ is a vector with each QoS-component as one dimension. The weight of power consumption depends on the current battery status, the weight of costs depends on the user preferences and is negative, and the weight of handover criteria depends on how sensitive to connection disruption an application is.

\section{DISCUSSION AND FUTURE RESEARCH TOPICS}

A full implementation of the presented concept is still problematic, particularly due to limitations in supporting vertical handovers on the terminal as well as on the network side. The technical capabilities of cross-technology handovers of both, the network and the terminal, must be taken into account in the process of prioritizing available connections. In case of exclusively horizontal handovers, available infrastructures and terminals already dispose of the required protocols for mobility support. For vertical handovers, there are several approaches under discussion but, to the best of our knowledge, there is no concept solving the handover problems for all available access technologies with acceptable delays. Furthermore, major problems are still caused by handover processes between networks of different operators. So for an efficient and universal implementation of the concept presented in this paper, advancements in vertical handover support across networks of different operators must be achieved. This topic is addressed by many research projects, e.g. by G-Lab [28].

\section{ACKNOWLEDGMENTS}

The concept presented in this paper has been developed within the research project G-Lab [28], a German future Internet research project related to Planet Lab and funded by the German Federal Ministry of Education and Research. 


\section{REFERENCES}

[1] Schneider, J., Mannweiler, C., Klein, A., Schotten, H.: Erfassung von Umgebungskontext und Kontextmanagement. 14. ITG Fachtagung Mobilkommunikation, Osnabrück (2009)

[2] Klein, A., Mannweiler, C., Schneider, J., Schotten, H.: A Framework for Intelligent Radio Network Access Based on Context Models. Proceedings of 22nd WWRF Meeting, Paris (2009)

[3] Taha, A.-E. M., Hassanein, H.S., Mouftah, H.T.: On Robust Allocation Policies in Wireless Heterogeneous Networks. Proceedings of the First International Conference on Quality of Service in Heterogeneous Wired/Wireless Networks (QSHINE'04) (2004)

[4] Yang, X., Bigham, J., Cuthbert, J.: Resource Management for Service Providers in Heterogeneous Wireless Networks. Wireless Communications and Networking Conference, New Orleans, LA (2005)

[5] Ormond, O., Perry, P., Murphy, J.: Network Selection Decision in Wireless Heterogeneous Networks. IEEE 16th International Symposium on Personal, Indoor and Mobile Radio Communications, Berlin (2005)

[6] Pawar, P., van Beijnum, B.-J., Wac, K., Hermens, H., Konstantas, D.: Towards Location Based QoS-Aware Network Selection Mechanism for the Nomadic Mobile Services. IEEE Consumer Communication and Networking Conference, Special Session on Beyond GPS - Where Navigation meets Consumer Communications (CCNC'09), Las Vegas, NV (2009)

[7] Blau, I., Wunder, G., Karla, I., Sigle, R.: Cost Based Heterogeneous Access Management in Multi-Service, Multi-System Scenarios. The 18th Annual IEEE International Symposium on Personal, Indoor and Mobile Radio Communications (PIMRC'07), Athens (2007)

[8] Hasswa, A., Nasser, N., Hassanein, H.: A seamless context-aware architecture for fourth generation wireless networks. Wireless Personal Communications, vol. 43, pp. 1035-1049. Springer, Heidelberg (2007)

[9] Chen, H.: An Intelligent Broker Architecture for Pervasive ContextAware Systems. University of Maryland, College Park, MD (2004)

[10] Want, R., Hopper, A., Falcao, V., Gibbons, J.: The active badge location system. ACM Transactions on Information Systems, vol. 10, pp. 91-102 (1992)

[11] Abowd, G.D., Atkeson, C.G., Hong, J., Long, S., Kooper, R., Pinkerton, M.: Cyberguide: a mobile context-aware tour guide. Wireless Networks 3(5), 421-433 (1997)

[12] Sumi, Y., Etani, T., Fels, S., Simonet, N., Kobayashi, K., Mase, K.: C-map: Building a context-aware mobile assistant for exhibition tours. In: Ishida, T. (ed.) Community Computing and Support Systems, Social Interaction in Networked Communities. LNCS, vol. 1519, pp.137-154. Springer, Heidelberg (1998)

[13] Cheverst, K., Davies, N., Mitchell, K., Friday, A., Efs-tratiou, C.: Developing a context-aware electronic tourist guide: some issues and experiences. In: Proceedings of the SIGCHI conference on Human Factors in Computing Systems, The Hague (2000)

[14] Jo, D., Lee, J., Lee, S., Ha, T., Kwon, T., Choi, Y.: Signal Dragging: Effects of Terminal Movement on War-Driving in CDMA/WCDMA Networks. In: Hightower, J., Schiele, B., Strang, T. (eds.) LoCA 2007. LNCS, vol. 4718, pp. 211-227. Springer, Heidelbeg (2007)

[15] Zhdanova, A.V., Zoric, J., Marengo, M., Van Kranenburg, H., Snoeck, N., Sutterer, M., Rck, C., Droegehorn, O., Arbanowski, S.: Context Acquisition, Representation and Employment in Mobile Service Platforms. 15th IST Mobile and Wireless Communications Summit, Myconos (2006)

[16] Floren, P., Przybilski, M., Nurmi, P., Koolwaaij, J., Tarlano, A., Wagner, M., Luther, M., Bataille, F., Boussard, M., Mrohs, B., Lau, S.: Toward a Context Management Framework for MobiLife. 14th IST Mobile and Communications Summit, Dresden (2005)

[17] Janneteau, C., Simoes, J., Antoniou, J., Christophorou, C., Kellil, M. Klein, A., Neto, A., Pinto, F.C., Roux, P. Sargento, S., Schotten, H.D., Schneider, J.: Context-aware Multiparty Networking. Conference Proceedings of ICT MobileSummit, Santander (2009)

[18] IEEE P802.21/D14, Draft Standard for Local and Metropolitan Area Networks: Media Independent Handover Services, New York (2009)

[19] Pils, C., Roussaki, I., Pfeifer, T., Liampotis, N., Kalatzis, N.: Federation and Sharing in the Context Marketplace. In: Hightower, J., Schiele, B., Strang, T. (eds.) LoCA 2007. LNCS, vol. 4718, pp. 121-138. Springer, Heidelberg (2007)

[20] Koh, S.J., Kim, S.W.: mSCTP for Vertical Handover Between Heterogeneous Networks. Conference Proceedings of 3rd International Conference on Human.Society@Internet, Tokyo (2005)

[21] Kim, K.R., Min, S.G., Han, Y.H.: Fast Handover Method for mSCTP Based Using FMIPv6. Conference Proceedings of International Conference on Mobile Ad-hoc and Sensor Networks, Wuhan (2005)
[22] Hasswa, A., Nasser, N., Hassanein, H.: A Seamless Context-Aware Architecture for Fourth Generation Wireless Networks. In: Wireless Personal Communications, vol. 43, pp. 1035-1049. Springer, Heidelberg (2007)

[23] Kim, D.S., Hong, C.S.: The Primary Path Selection Algorithm for Ubiquitous Multi-homing Environments. In: S. Ata and C.S. Hong (Eds.): APNOMS 2007, LNCS 4773, pp. 296305. Springer, Heidelberg (2007)

[24] Ma, L., Yu, F., Leung, V.C.M., Randhawa, T.: A New Method to Support UMTS/WLAN Vertical Handover Using SCTP. In: IEEE Wireless Communication, vol. 11, issue 4, pp.44-51 (2004)

[25] Menth, M., Hartman, M., Tran-Gia, P., Klein, D.: Future Internet Routing: Motivation and Design Issues. In: Information Technology, vol 50, issue 6, pp. 358-375. Oldenburg Wissenschaftsverlag (2008)

[26] Asadullah, S., Mahmoud, A.S., Abu-Amara, M., Sheltami, T.: Vertical Handoff Characterization for SIP and mSCTP Based UMTS-WLAN Integration Solutions.In: IEEE GCC, pp.11-14 November, Manama, Bahrain (2007)

[27] Stewart, R., Xie, Q., Tuexen, M., Maruyama, S., Kozuka, M.: Stream Control Transmission Protocol (SCTP) Dynamic Address Reconfiguration. IETF Draft, draft-ietf-tsvwg-addip-sctp-17 (November 2006)

[28] www.german-lab.de 